\title{
A Large-Scale Study of Changes to the Quantity, Quality, and Distribution of Video Game Play During a Global Health Pandemic
}

\author{
Matti Vuorre ${ }^{1}$, David Zendle ${ }^{2}$, Elena Petrovskaya ${ }^{2}$, Nick Ballou ${ }^{3}$, and Andrew K. Przybylski ${ }^{1,4}$ \\ ${ }^{1}$ Oxford Internet Institute, University of Oxford \\ ${ }^{2}$ Department of Computer Science, University of York \\ ${ }^{3}$ Game AI and Cognitive Sciences Groups, Queen Mary University of London \\ ${ }^{4}$ Department of Experimental Psychology, University of Oxford
}

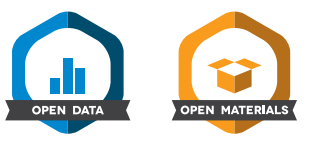

\begin{abstract}
Video game play has been framed as both protective factor and risk to mental health during the Coronavirus disease (COVID-19) pandemic. We conducted a statistical analysis of changes to video game play during the pandemic to better understand gaming behavior and in doing so provide an empirical foundation to the fractured discourse surrounding play and mental health. Analyses of millions of players' engagement with the 500 globally most popular games on the Steam platform indicated that the quantity of play had dramatically increased during key points of the pandemic; that those increases were more prominent for multiplayer games, suggesting that gamers were seeking out the social affordances of video game play; and that play had become more equally distributed across days of the week, suggesting increased merging of leisure activities with work and school activities. These results provide a starting point for empirically grounded discussions on video games during the pandemic, their uses, and potential effects.
\end{abstract}

Keywords: video games, COVID-19, technology

Supplemental materials: https://doi.org/10.1037/tmb0000048.supp

The declaration of Coronavirus disease (COVID-19) as a global pandemic by the World Health Organization on March 11th $2020^{1}$ preceded important changes to the lives of people across the world. National lockdowns and local stay-at-home orders limited behaviors in a number of ways ranging from decreased mobility to disruptions to leisure, social, and civic activities (Bonaccorsi et al., 2020; García et al., 2020; Rudnicka et al., 2020). Conversely, other pursuits saw increased interest as providers of "stay-at-home" tools for work, education, and leisure such as Netflix, Peloton, and Zoom substantially increased in engagement and valuation as the pandemic, and the global response to it, took hold. Just as the activities that people

\footnotetext{
${ }^{1}$ https://www.who.int/director-general/speeches/detail/who-director-generals-opening-remarks-at-the-media-briefing-on-covid-19---11-march-2020
}

Action Editor: C. Shawn Green was the action editor for this article. ORCID Ids: Matti Vuorre (iD) https://orcid.org/0000-0001-5052-066X; David Zendle (iD) https://orcid.org/0000-0003-0279-6439; Elena Petrovskaya (D) https://orcid.org/0000-0003-4276-6154; Nick Ballou (D) https://orcid.org/ 0000-0003-4126-0696; Andrew K. Przybylski (DD https://orcid.org/00000001-5547-2185.

Acknowledgements: We are grateful to Pavel Djundik (SteamDB) for assistance in collecting the data.

Funding: This research was supported by the Huo Family Foundation.

Disclosures: The authors declare no conflicts of interest.

Author Contributions: Conceptualization: Matti Vuorre, David Zendle, Elena Petrovskaya, Nick Ballou and Andrew K. Przybylski. Data Curation: Matti Vuorre and David Zendle. Formal Analysis: Matti Vuorre. Funding Acquisition: Andrew K. Przybylski. Investigation: Matti Vuorre and David Zendle. Methodology: Matti Vuorre, David Zendle, Elena Petrovskaya, Nick Ballou and Andrew K. Przybylski. Project Administration: Matti Vuorre, David Zendle and Andrew K. Przybylski. Resources: Andrew K. Przybylski. Software: Matti Vuorre and David Zendle. Supervision: Andrew K. Przybylski. Validation: Matti Vuorre. Visualization: Matti Vuorre.
Writing of Original Draft Preparation: Matti Vuorre, David Zendle and Andrew K. Przybylski. Writing of Review and Editing: Matti Vuorre, David Zendle, Elena Petrovskaya, Nick Ballou and Andrew K. Przybylski.

Data Availability Statement: The raw data and annotated analysis code supporting this work are available at https://osf.io/ya9jt/. A public preprint of this work is available at https://psyarxiv.com/8me6p/

Open Science Disclosures:

Ait The data are available at https://osf.io/n $7 \mathrm{mzv}$

3 The experiment materials are available at https://osf.io/n7mzv

Open Access License: This work is licensed under a Creative Commons Attribution-NonCommercial-NoDerivatives 4.0 International License (CC-BYNC-ND). This license permits copying and redistributing the work in any medium or format for noncommercial use provided the original authors and source are credited and a link to the license is included in attribution. No derivative works are permitted under this license.

Contact Information: Correspondence concerning this article should be addressed to Andrew K. Przybylski, Oxford Internet Institute, University of Oxford, 1 St Giles, Oxford OX1 3JS, United Kingdom. Email: andy.przybylski@oii.ox.ac.uk 
are engaging in appear to have shifted during the pandemic, so too has when people do things, due to changes in living conditions and other disruptions caused by the pandemic. Although the research literature on this topic is nascent, the proposed effects range from changes in sleep patterns to a greater incorporation of family and leisure time into the working day (Raabe et al., 2020; Rome et al., 2021; Rudnicka et al., 2020).

Because of their interactive nature and social affordances, video games are a domain that we would expect to be affected by the pandemic, and the individual and societal responses to it. Indeed, school closures may have led young people to have more time for video games, and the redistribution of adults' working lives, unmoored from commuting and face-to-face meetings on weekdays, may have enabled individuals to play games during times when they might otherwise be occupied (Amin et al., 2020). Shifts in these patterns of play are plausible, but research concerned with this idea is sparse and predominated by self-report data and samples of convenience, leading to potentially inaccurate results that do not generalize well to broader populations. For example, participants in one study of 465 individuals exposed to a COVID-19 lockdown in Italy reported playing more multiplayer video games during lockdown than they had before (Gabbiadini et al., 2020). In another survey, 78 gamers recruited from online platforms such as Twitter and Reddit reported playing more multiplayer video games during lockdown than they had before (Cmentowski \& Krüger, 2020). Survey data from one larger, nationally stratified sample, suggest that there might have been a slight uptick in video game play, about 12 min a day on average, in April 2020 during the second week of the U.K. lockdown, but that shift quickly returned to baseline (Creative Industries Policy \& Evidence Centre, 2020). Such work lends general support to our intuition of what might have happened to patterns of video game play during the pandemic, but also that actual changes might have been too small to merit discussion. However, because self-report measures of digital activities such as game play are known to be unreliable (Johannes et al., 2021; Parry et al., 2021), and because results from convenience samples are unlikely to generalize directly to the broader populations, we actually do not know whether these isolated data points reflect true (small) upward global trends in the quantity of video game play, or whether they are artifacts of sampling protocols or self-report biases.

Beyond quantity of play, even less is known about the types and qualities of the games which were played during the height of the pandemic. This oversight is important, because modern online video games such as Fortnite, Minecraft, and Call of Duty afford multiple channels of socializing and virtual venues for connecting, collaborating, and playing together, all of which might be important when interactions in person are made more difficult. Marston and Kowert (2020, p. 5) proposed that games provide a "useful tool for mitigating some of the negative impacts of COVID-19 for adults" because of the benefits of in-game socialization to "[reduce] stress, depression, and sense of loneliness." A range of similar claims about the social benefits of games have been made by others who have observed that "games can help cope with the negative effects of social isolation ... multiplayer games are known to strengthen the connectedness with a virtual community, which could benefit the overall mental health by reducing feelings of solitude" (Cmentowski \& Krüger, 2020, p. 2). On the basis of nearly 2 decades of research on video games which shows that they can provide rich opportunities for players to meet their fundamental psychological needs, including feeling a sense of belonging, we would expect that this could be true (Orben et al., 2020). That said, there is little evidence that these games in particular are seeing an outsized increase in engagement vs. other forms of video game play, although the idea that there have been significant increases in social video game play, and that such play might be beneficial to wellbeing, has taken hold in popular discourse (Baraniuk, 2020; Fishman, 2020; Stieg, 2020).

This exuberance about the potential positive aspects of games stands in contrast to similarly earnest warnings that increased video game play during lockdown is a harbinger of games-related problems. Indeed, some have proposed that COVID-19 provides a dangerous mix of home confinement, stress, fear, and uncertaintyand that this is likely to lead to the excessive use of "reinforcing behaviours" such as video games (Király et al., 2020, p. 2). Similarly, Ko and Yen $(2020$, p. 2) posit the stressful nature of the pandemic, coupled with the high relative availability of video games, make it likely that the "maladaptive use of gaming has become more frequent during the COVID-19 outbreak because many alternative [coping] strategies have become impracticable." Yet, others have proposed that the prolonged lack of structure arising from school closures may leave children and adolescents more susceptible to "loneliness, addiction to videogames and binge watching" (Poletti \& Raballo, 2020, p. 1). Going further, (King et al., 2020, p. 2) assert that "significant increases in gaming ... may pose risks for vulnerable individuals including minors and those affected by and at risk of gaming disorder."

Taken together, these warnings can be understood as speculative applications of diathesis stress models, whereby COVID-19-related stressors drive vulnerable individuals towards increasingly harmful patterns of behavior, including video game play, to the extent that it further interferes with healthy functioning. Taking for granted that increases in play have negative effects and that increases in engagement are in evidence, one might assume the combined pressures of the pandemic and steps to remediate it amplify health risks assumed to be associated with video games. However, as with studies suggesting potential benefits of games during the COVID-19 pandemic, the literature focusing on their potential negative impacts has also exclusively used self-report methodologies in addition to convenience samples to their potential detriment (see also Li et al., 2021; Teng et al., 2021).

Taken as a whole, it is clear that the rhetoric and discourses surrounding video games during the pandemic have outstripped what we empirically understand about the ways human play behavior shifted, or not, during 2020. On the basis of anecdote and common sense, it is plausible that various changes in engagement behavior may have occurred during the COVID-19 pandemic, but the scale, time course, and lasting impacts on play behavior are unknown. Leisure behaviors such as video game play are known to have temporal cycles, such as the regular increase in video game play on weekends, and these patterns may have been disrupted or indeed magnified: COVID-19-related changes to patterns in work and the restructuring of school and family time may have increased the accessibility of games during the week, shifting these cycles and freeing up more time for play, thus restructuring how individuals spent their time. In addition, there have likely been changes to the qualities of the games played; specifically, their social affordances in the form of multiplayer features may have experienced outsized 
popularity during this period. Although large-scale research using actual game play data has found that time spent playing games such as Animal Crossing: New Horizons and Plants vs. Zombies: Battle for Neighborville is slightly but positively correlated with wellbeing (Johannes et al., 2021), it remains unclear if play has actually increased to a degree that should encourage those excited about positive aspects of game play, or worry those concerned about possible harms associated with play.

\section{The Present Study}

Our goal in this study was to examine how video game play behavior changed during the COVID-19 pandemic and in doing so provide an empirically grounded perspective to inform the fractured discourse surrounding the assumptions that are being made about changes to gaming behaviors and their health effects during this period. This study does not directly address mental health; instead, we are interested in changes to the base rate of gaming behavior and argue that this is a necessary datum to be defined before proceeding with more fine-grained investigations of what the changes might mean psychologically.

To this end, we aggregated and analyzed data on a diverse range of the 500 globally most popular games that played on the Steam online games platform. ${ }^{2}$ Our aims for analyzing data generated by several millions of daily players across a wide range of popular video game titles were threefold. First, we wanted to derive an estimate of the quantity of change, if any, in video game play during the pandemic. Second, we wanted to evaluate if any changes in play depended on the differences between single- and multiplayer games. Finally, we examined whether the pandemic influenced the cyclical nature of play, changing the distribution of game engagement across the week.

\section{Method}

We gathered the data that analyzed in this study from the SteamDB website, ${ }^{3}$ which interfaces with Steam's application programming interface (API) and records the peak number of simultaneous players for each game on the Steam platform on each day. Although these data originate from the official Steam API, some aspects of video game play on the platform may be absent from the data set. For example, players who do not have an active Internet connection during play are not counted, nor do those playing certain downloadable content (DLC) expansions that are listed separately from the original game count in the player tally of the original game. The scope of the data set is global: Players may be located anywhere in the world, but the times on SteamDB are recorded in UTC \pm 00:00. This means that, for example, a player on the American west coast playing a game on a Friday night may be counted towards the peak number of players on a Saturday.

The full data set consists of 40,009 games and begins Jan 1, 2000. To limit the scope of the manual coding while retaining the most played recent games, we elected to analyze the top 500 games based on their mean peak daily players during 2019. We reached this decision, because the distribution of players was heavily rightskewed, with the top 500 games of 2019 accounting for $91.3 \%$ of the total peak player counts during the year. Therefore, coding additional games would not have substantially increased the scope of the data (games 501-1,000 account for just an additional $2.8 \%$ of players).

Prior to analysis, we content-coded these 500 games in terms of their social affordances, specifically as either single player or multiplayer, defined as containing a feature which allows more than one player to remotely interact with the same game environment via the Internet in a substantial way. We coded the games as single- or multiplayer based on whether the features were present in the game at the time of analysis, given that some games had added or removed features because of their release (Zendle et al., 2020). We considered a game only to have a feature if it was included in the game itself, not any additional DLCs or user-generated modifications.

Two coders independently assessed the list of 500 games for the above features, having had a discussion about the above definition and how it might be implemented in practice prior to beginning the coding. The assessments drew on a variety of sources, such as the original game documentation, game-specific wikis, and forum posts and other player discussions. If a feature was difficult to identify, game play videos were also watched, and in the most extreme cases, the games were downloaded and played.

Cohen's Kappa measures the degree of agreement between coders to account for chance agreement, and is the most commonly used way to measure intercoder reliability. A Kappa statistic of greater than or equal to 0.81 is classed as being "almost perfect agreement" (McHugh, 2012, p. 4). For this reason, to keep consistency with our previous work (Zendle et al., 2020), we set a minimum threshold of agreement of 0.81 . Any remaining disagreements would be resolved through discussion, so that the final data set would be fully reflective of the opinions of both coders, to ensure maximum validity.

After the first round of coding, the kappa scores for the presence of multiplayer features were 0.68 . Given that this score was lower than our target of 0.8 , the coders discussed and revisited elements of the definitions which may have caused confusion, landing on a clarification that multiplayer games contain "a feature that allows more than one player to remotely interact with the same game environment via the internet in a substantial way," and that this definition refers specifically to shipped versions of games, rather than updates via community modification or third-party tool use (e.g., Parsec).

The two coders then separately revisited and recoded the games on which there was disagreement in a second pass. After the second pass, the required threshold of a Kappa of 0.8 was met $(k=0.85)$. However, there remained a total of 21 games on which there was a coder disagreement. The two coders held a meeting in which they resolved any remaining disagreements through discussion. The majority of conflicts arose due to initial misinterpretation of community-modified game content as being a part of the official game, or lack of realization that additional game features had been added or removed because of their release. In the end, perfect agreement was reached for all 500 games.

The final data set consisted of 500 games'-manually coded for single- or multi-player-peak daily concurrent players on the Steam platform from January 1st 2019 to December 31st 2020. Because we

\footnotetext{
${ }^{2}$ https://store.steampowered.com/

${ }^{3} \mathrm{https}: / /$ steamdb.info. It should be noted that SteamDB does not commonly allow crawling of their database, but allows exceptions for academic research. We applied for and were granted such an exception.
} 
were interested in the presence or absence of social affordances, not the individual games themselves, the data set was collapsed to total daily counts for single- and multi-player games.

\section{Analytic Approach}

In line with the three research questions guiding this study, we analyzed the daily Steam engagement data in terms of COVID-19related changes to three features reflecting the quantity, quality, and distribution of play across the week. However, because the player counts are at a global level, they cannot be directly linked to local health advisories, and consequently we did not use specific COVID19 advisory time periods as predictors of video game play or changes therein. Instead, in addition to visualizations, we modeled daily Steam player counts with a Generalized Additive Model (GAM) using the mgcv package in R (R Core Team, 2019; Wood, 2017, 2020), with the main comparison being changes in video game play patterns between 2019 and 2020. We included week (0-52) and weekday (0-6) as predictors of the daily player count to describe changes in play over time within the year (week predictor) and week (weekday predictor). The week and weekday predictors were modeled using tensor product smooths, with cubic regression splines, such that the time course of the player count could take any potentially nonlinear shape as indicated by the data, and thus accurately describe any changes to the quantity of video game play over time. The smooth functions were estimated separately for years (2019 and 2020) and game types (multi- and single player) to allow comparisons between years and game types. In addition, the tensor product interaction between week and weekday allowed the differences between weekdays to change in potentially nonlinear manners over the course of the year. Thus, the model facilitated our examination of the degree to which the quantity of play, social quality of play, and its weekly distribution changed over time.

\section{Data and Code Availability}

The raw data and annotated analysis code are available on the Open Science Framework (Vuorre et al., 2020).

\section{Results}

We first focused on changes to the quantity of play behaviors during the COVID-19 pandemic. Observing the daily number of players in 2019 and 2020 (shown separately in the top row of Figure 1 for multi- and single player games), it is clear that the volume of players in 2020 increased rapidly in mid-March, peaked in early April, and then slowly declined to levels comparable with January and February by late June. Following this, there was a slow increase in engagement beginning around October 2020. Relative to these changes, player numbers in 2019 remained stable throughout

\section{Figure 1}

Top Row: Daily Total Players (in Millions) of the Top 500 Video Games on the Steam Platform (Points), and Generalized Additive Model Estimates in 2020 (Black) and 2019 (Light Grey)
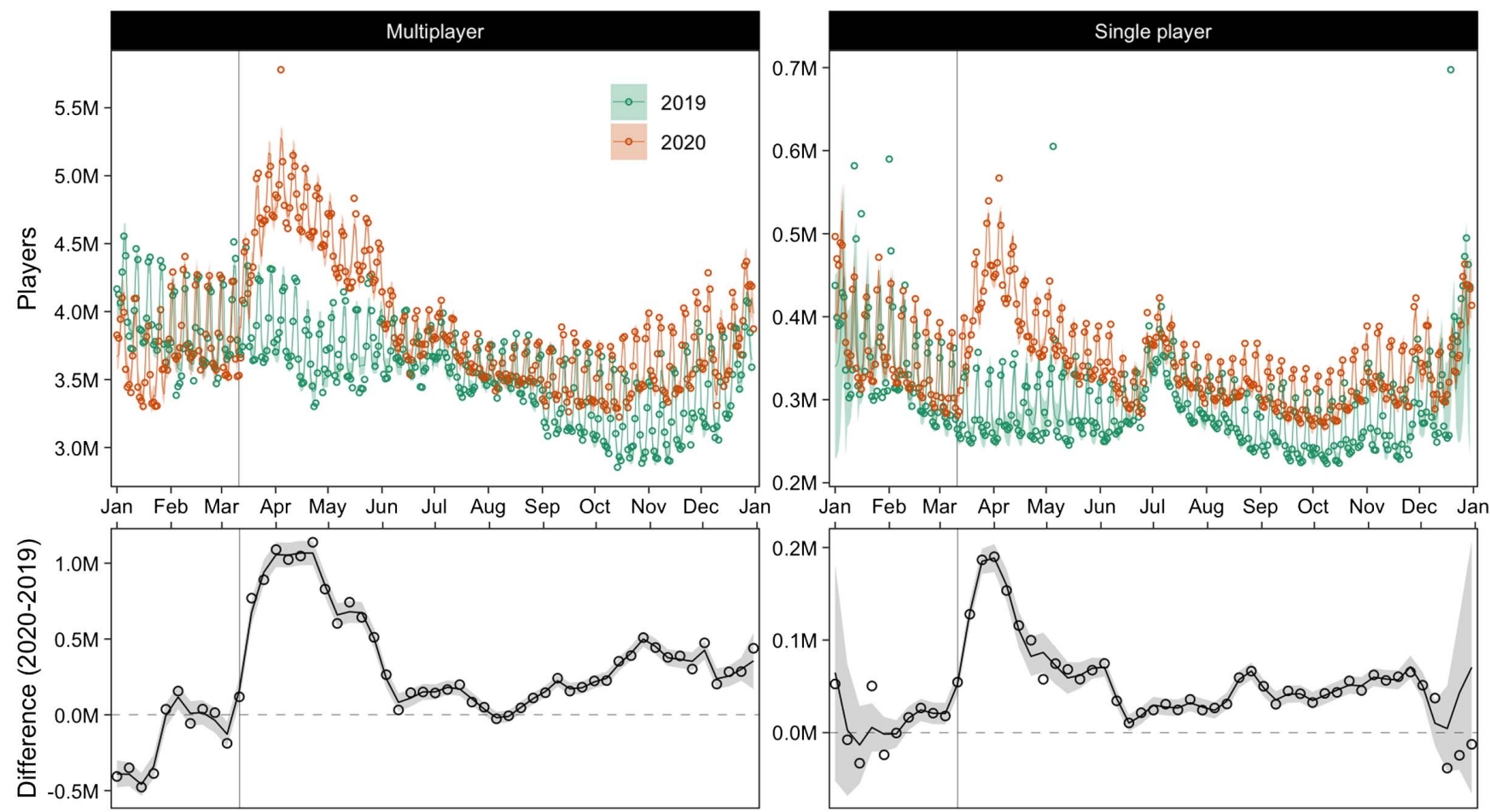

Note. Lines and shades indicate model fits with 95\% CIs. The vertical line indicates March 11th when the WHO declared COVID-19 a pandemic. Bottom row: Differences in the average weekly player counts in 2019 and 2020 (2019 weekly averages subtracted from 2020 weekly averages). 
Figure 2

Top Panel: Difference Between the Number of Players Engaged with Multi- and Single Player Games for Each Week in 2019 (Green) and 2020 (Red)
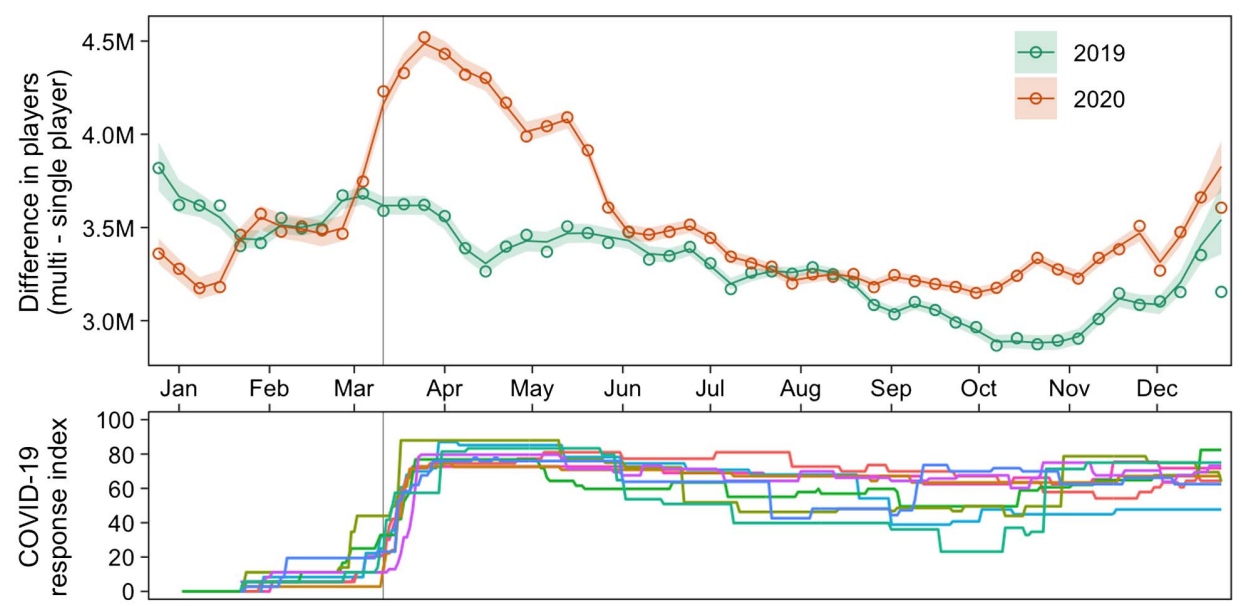

$$
\begin{aligned}
& \text { - Brazil - France - Poland - Turkey United States } \\
& \text { - Canada - Germany - Russia - United Kingdom }
\end{aligned}
$$

Note. Points are observed differences between the weekly multiplayer and single player counts (weekly means), lines and shades are model-implied differences and 95\% CIs. Bottom panel: Index of the stringency of nine nations' response to the COVID-19 pandemic (Hale et al., 2020).

the year. These observations supported increased games engagement during the early phases of the COVID-19 pandemic, and a slow return to comparably standard levels of games engagement when initial lockdowns and advisories were eased in much of the world in the (northern hemisphere) summer (see Figure 2, bottom panel).

To clarify these observed differences, the bottom row of Figure 1 shows the difference in average weekly game engagement between 2019 and 2020. It is clear that, following the WHO announcement in March 2020, there was a very large increase in play in 2020, observed for both single- and multiplayer games, reaching as many as $1.14 \mathrm{M}$ and $0.19 \mathrm{M}$ excess multi- and single players in April 2020, respectively, and a more modest increase after the relatively standard levels of play seen in June to August.

Second, we compared changes to the quality of play, contrasting the popularity of multi- and single player games, over time in 2019 and 2020. Figure 2 (top panel) shows the difference between player numbers for these two game types for each week in 2019 and 2020. In addition, Figure 2 (bottom panel) shows the stringency index of the national response to COVID-19 for nine nations that accounted for the majority of Steam traffic in $2018^{4}$ (Guidotti \& Ardia, 2020; Hale et al., 2020), a measure of the degree to which life was restricted in response to the pandemic in those countries, to allow comparing changes in the game engagement data (top panel) to responses to the pandemic (bottom panel). Concurrent with the changes to the volume of play described above, and to the changes in COVID-19 responses shown in Figure 2 (bottom), beginning in late March 2020, there was a large increase in the popularity of multiplayer games over single player games. The difference between multi- and single player players reached 4.38 million players in April 2020, in contrast to a difference of only 3.52M in February 2020. No such trend was observed for
2019 when the difference between single- and multiplayer games had remained relatively more stable (February: 3.45M; April: 3.46M), suggesting that the increased popularity of multiplayer games in 2020 (2nd quarter) was at least partly driven by COVID-19 responses.

Third, concerning the distribution of play across week, we investigated the extent to which the weekend effect-the greatly increased amount of video game play on weekends-had changed over time. The weekend effect is salient as the sawtooth pattern in Figure 1; to illustrate it in more detail, the top row of Figure 3 shows the amount of play for each day of the week for a select number of weeks. As shown, game engagement in early 2020 and 2019 was relatively stable from Monday to Thursday, with a small increase on Friday, and a pronounced increase on Saturday and Sunday. The middle row of Figure 3 displays the magnitude of this weekend effect (weekend-weekday engagement) over time in 2019 and 2020. Towards the middle of the year, the weekday effect diminished in both 2019 and 2020, but even more so in 2020. The bottom row of Figure 3 shows the difference in the weekend effect between 2019 and 2020: Even though the yearly trend, whereby the weekend effect was smaller in the middle of the year was observed both in 2019 and 2020, the difference between years shows that, broadly following March 11th, the weekend effect was and remained notably smaller in 2020 as compared to 2019, an effect observed for both single- and multiplayer games. As an example, the average weekend effect in April 2019 was $0.19 \mathrm{M}$, but in April 2020 only $0.11 \mathrm{M}$-almost $60 \%$ smaller. That is, when people played had become more evenly

\footnotetext{
${ }^{4}$ https://www.statista.com/statistics/826870/steam-distribution-country/; we left out China even though it was in the top-10 that year, because Steam was not officially available there until 2021.
} 
Figure 3

Differences in the Distribution of Play Across the Week
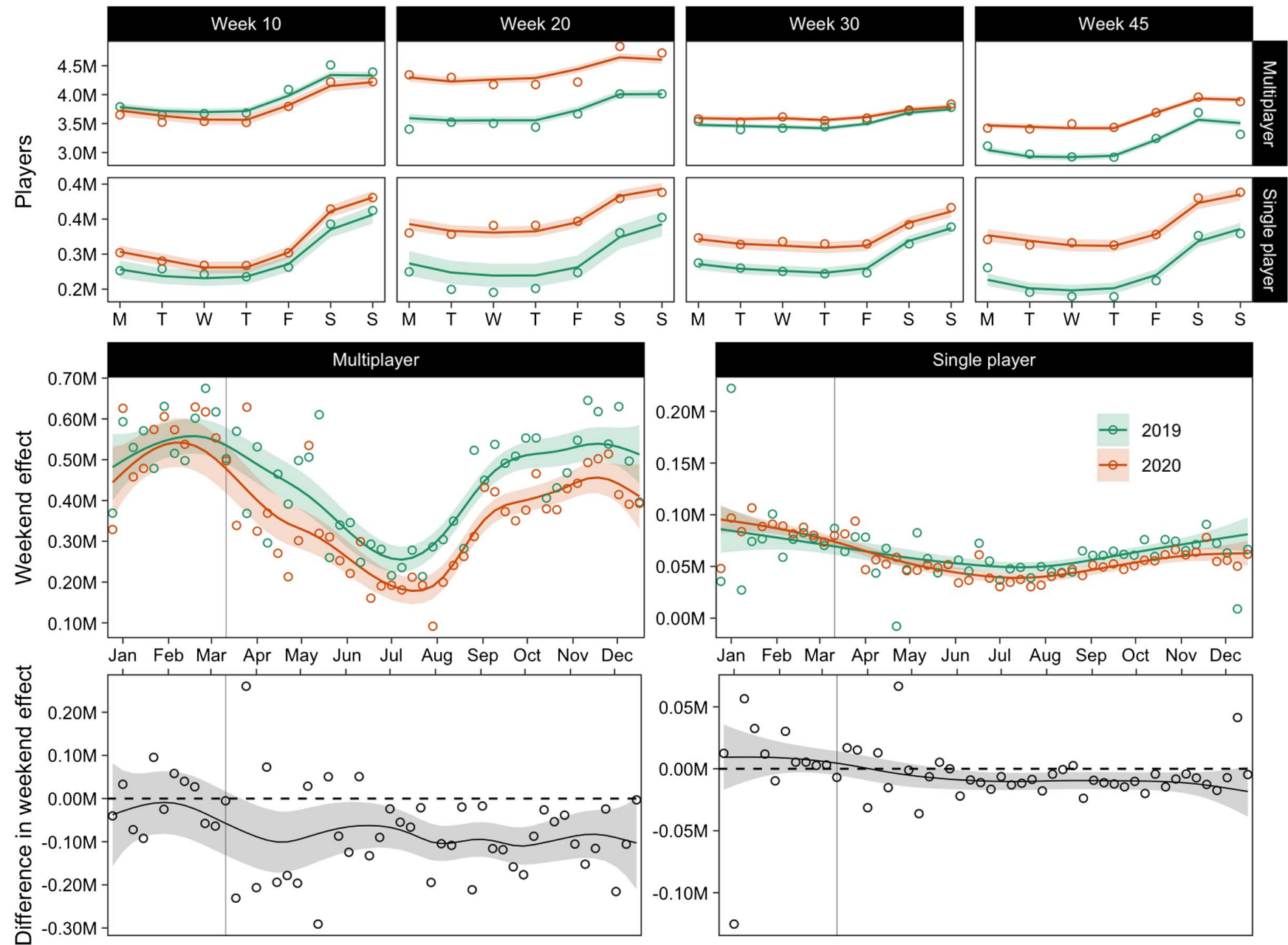

Note. Top: Number of players on each weekday for a sample of illustrative weeks (columns), separately for single- and multiplayer games (two rows). Points indicate observed data; lines and shades are GAM fits with 95\%CIs. Middle: The weekend effect (mean of weekday players subtracted from the mean of Saturday and Sunday players) separately for 2019 (green) and 2020 (red). Bottom: Difference in the weekend effect (2020-2019).

distributed throughout the week during the 2020 COVID-19 pandemic, such that the difference between weekend and weekday engagement was smaller. This observation suggests that COVID19-related changes might have driven changes in patterns of behavior, allowing for (relatively) more video game play time during the week.

\section{Discussion}

Speculation in the media and among researchers about the impacts of the COVID-19 pandemic on video game play behavior and its lasting effects on players is rife (Cmentowski \& Krüger, 2020; Gabbiadini et al., 2020; Király et al., 2020; Ko \& Yen, 2020). However, large-scale and representative examinations of actual changes to game behavior have not yet been conducted. The results of our study, based on data from millions of players from the popular Steam platform, provide evidence that video game behavior did in fact substantively increase during the pandemic. The number of people engaging with the top 500 games on Steam remained relatively stable from the beginning of March 2019 to the end of June of 2019 (ranging from $0.28 \mathrm{M}$ to $0.30 \mathrm{M}$ players for single player, and 3.70 to 3.94 players for multiplayer games). However, during 2020, a new and distinct pattern of behavior emerged: A sharp increase in play of both single-player and multiplayer games after the WHO pandemic declaration on March 11th, with player numbers ranging from $0.33 \mathrm{M}$ to $0.42 \mathrm{M}$ for single player and $3.85 \mathrm{M}$ to $4.80 \mathrm{M}$ for multiplayer in the months of March to June. These changes were broadly in line with fluctuations in the stringency of governments' responses to the pandemic, as observed in Figure 2 (Hale et al., 2020). This suggests that global changes in the behavior of individuals associated with the COVID-19 pandemic did indeed have marked short-term effects on games engagement. Interestingly, our results also suggested a return to regular overall video game engagement volumes in July of 2020 and a slower increase beginning in October. At least in terms of the overall volume of play, this 
evidence appears to run counter to the idea that the pandemic unlocked prolonged "significant increases in gaming" as some have feared (King et al., 2020). However, it should be noted that the data analyzed here is aggregated at the level of individual games: While it may suggest a return to prepandemic levels of overall gaming after July of 2020, it cannot speak to changes in the patterns of gaming amongst individuals. For example, such a regression may be the consequence of an influx (and outflux) of new gamers. It may well be the case that overall increases in gaming within individuals are masked by this level of data aggregation. Furthermore, the broader importance of this result is unclear, as it is evidence that play, along with other behaviors, returned to at least partially regular patterns of daily life after initial lockdowns and stay-at-home orders ceased to be in effect.

Building on these observations, our analyses indicated that the social affordances of games, namely, whether they were single-player or multiplayer, accounted for much of these differences (Figure 2). Indeed, the peak number of daily players of single-player games in April 2020 was $0.57 \mathrm{M}$ and $5.78 \mathrm{M}$ for multiplayer games, in comparison with only $0.36 \mathrm{M}$ and $4.24 \mathrm{M}$, respectively, in April 2019. Critically, although multiplayer games were vastly more popular in 2019 than were single player games, this difference increased even further beginning in March of 2020, and again starting in September 2020, following a brief period of differences comparable to 2019 in the northern hemisphere summer months (Figure 2).

One interpretation of these data is that the relative uplift in multiplayer games may relate to their ability to satisfy basic psychological needs (e.g., Ryan et al., 2006). The increased gap in the volume of gaming behavior between multiplayer and single player games following March 2020 suggests that in-game affordances which support the psychological needs for relatedness, and a sense of social belonging, might be key to driving increased gaming behavior during the most restrictive periods of the pandemic. However, caution is warranted in this interpretation of the data: While a divergence in growth between multiplayer and single player games was observed, it is unclear whether this shift may be the product of some third factor. Other plausible explanations, for example, are that multiplayer games may be less expensive, or tend to afford longer amounts of play before completion. Our data cannot speak to the motivations for shifts in play, or the consequences of these shifts in play. It can merely describe how play changed during the COVID-19 pandemic.

The pandemic has also disrupted how people structure and distribute their work, leisure, education, and family time across the week (Rome et al., 2021; Rudnicka et al., 2020). Our results highlighted the degree to which this disruption is reflected in video game play. Figure 1 indicated a cyclical pattern in the number of play sessions on Steam through 2019 and 2020, with more players engaging on Saturdays and Sundays than on other weekdays. ${ }^{5}$ In other words, more play occurs during the weekend. However, there were important changes to this pattern following March 2020: When one considers February of both 2019 and 2020, there is a similar weekly pattern of play occurring in both cases: For example, there were average $3.80 \mathrm{M}(2020: 3.83 \mathrm{M})$ million daily multiplayers on Monday-Friday, and approximately 4.21M (2020: 4.31M) million daily multiplayers on Saturday and Sunday. By April of each year, however, these patterns had diverged. In April 2019, the average weekday saw $3.71 \mathrm{M}$ multiplayers, and the average weekend $4.03 \mathrm{M}$ multiplayers, for a difference of $0.32 \mathrm{M}$. In April of 2020, the average weekday multiplayer number was $4.81 \mathrm{M}$, with only a $0.17 \mathrm{M}$ increase to $4.98 \mathrm{M}$ on weekends. Crucially, weekly patterns of game play appeared to change during the pandemic: The relative boosts in engagement associated with the weekend had diminished during the pandemic and continued to be smaller throughout 2020, an effect observed for both single- and multiplayer games, but of greater magnitude for the latter (see Figure 3, bottom panel).

Keeping these findings in mind, their implications are qualified in at least three important ways. First, these data map the daily peak number of players of the 500 most popular games on Steam (in 2019). Although Steam is the predominate platform for computerbased games, it is only one of many online games platforms and cannot, therefore, provide insights into play engaged on mobile phones, tablets, and game consoles. For example, some consolespecific games, such as Animal Crossings: New Horizons, were immensely popular during the pandemic. Increased engagement with leisure activities is also likely to not be specific to video games but also, for example, watching streaming content. Second, the data that we analyzed captured play behavior at a global level of analysis in terms of peak daily users and cannot speak to the specific actions happening within games. For example, we coded the game CounterStrike: Global Offensive, a team-based first-person shooter, as a multiplayer game. We infer increases in engagement with this game as evidence of increased social game engagement, at least relative to single-player games which afford no such modes of in-game social engagement. Making this assumption might overestimate social play, because sessions of multiplayer game can be solitary (e.g., commerce) or underestimate it, because game-related socializing happens on other platforms (e.g., Discord). Finally, the data that we present provide a foundation for empirically grounded discussion on the impact of gaming during the pandemic on health, but our data are not sufficiently granular to test them. We simply do not currently have access to the kinds of detailed individual-level longitudinal data to assess such impacts. To study video game play, and how it impacts players, with the necessary level of accuracy and detail, we see large-scale transparent collaborations with the video game industry as a key path forward (Johannes et al., 2021).

This study provided an empirical starting point for discussions surrounding how video game play changed during the global pandemic. Our analysis makes clear that video game play increased, these shifts were driven mainly by games which provided players social opportunities, that play became more evenly distributed across the week, and that most of these changes to play were short-lived. These observations about changes to game play behaviors provide a foundation to discussions about potential benefits and harms of video games, and about how the pandemic may have impacted player health through changes to video game play behaviors. These findings underscore the value of objective (vs. self-reported) behavioral data and underscore the need for transparent collaborations between independent and video game industry data scientists. It is indeed possible that online play and socializing do materially influence health during a global pandemic, but it will not be possible to know if, or to what degree, this is true without a step change in both scientific transparency and data access.

\footnotetext{
${ }^{5}$ The diminished weekend effect in mid-year, for both 2019 and 2020, but more so in 2020, might reflect the fact that the majority of Steam engagement comes from the Northern hemisphere, where generally school breaks and holidays occur during this time.
} 


\section{References}

Amin, K. P., Griffiths, M. D., \& Dsouza, D. D. (2020). Online gaming during the COVID-19 pandemic in India: Strategies for work-life balance. International Journal of Mental Health and Addiction. https://doi.org/ 10.1007/s11469-020-00358-1

Baraniuk, C. (2020, April 13). Computer games: More than a lockdown distraction. BBC News. https://www.bbc.com/news/business-52210938

Bonaccorsi, G., Pierri, F., Cinelli, M., Flori, A., Galeazzi, A., Porcelli, F., Schmidt, A. L., Valensise, C. M., Scala, A., Quattrociocchi, W., \& Pammolli, F. (2020). Economic and social consequences of human mobility restrictions under COVID-19. Proceedings of the National Academy of Sciences of the United States of America, 117(27), 1553015535. https://doi.org/10.1073/pnas.2007658117

Cmentowski, S., \& Krüger, J. (2020). Playing with friends-The importance of social play during the COVID-19 pandemic [Conference session]. Extended Abstracts of the 2020 Annual Symposium on Computer-Human Interaction in Play, New York, United States. https://doi.org/10.1145/ 3383668.3419911

Creative Industries Policy and Evidence Centre. (2020). Digital culture: Consumption in lockdown. https://pec.ac.uk/assets/images/The-PECand-the-IPO-cultural-consumption-study-insights-from-the-six-weekstudy.pdf

Fishman, A. (2020, March 23). Why I bought a video game to cope with Coronavirus. Psychology Today. https://www.psychologytoday.com/blog/ video-game-health/202003/why-i-bought-video-game-cope-coronavirus

Gabbiadini, A., Baldissarri, C., Durante, F., Valtorta, R. R., De Rosa, M., \& Gallucci, M. (2020). Together apart: The Mitigating role of digital communication technologies on negative affect during the COVID-19 outbreak in Italy. Frontiers in Psychology, 11, Article 554678. https:// doi.org/10.3389/fpsyg.2020.554678

García, R., Gayer, C., Hölzl, W., Payo, S., Reuter, A., \& Wohlrabe, K. (2020). The impact of the COVID-19 crisis on European businesses: Evidence from surveys in Austria, Germany and Spain (Research Report No. 31). EconPol Policy Brief. https://www.econstor.eu/handle/10419/221818

Guidotti, E., \& Ardia, D. (2020). COVID-19 data hub. Journal of Open Source Software, 5(51), Article 2376. https://doi.org/10.21105/joss.02376

Hale, T., Angrist, N., Boby, T., Cameron-Blake, E., Hallas, L., Kira, B., Majumdar, S., Petherick, A., Phillips, T., Tatlow, H., \& Webster, S. (2020). Oxford COVID-19 government response tracker. Blavatnik School of Government.

Johannes, N., Vuorre, M., \& Przybylski, A. K. (2021). Video game play is positively correlated with well-being. Royal Society Open Science, 8(2). https://doi.org/10.1098/rsos.202049

King, D. L., Delfabbro, P. H., Billieux, J., \& Potenza, M. N. (2020). Problematic online gaming and the COVID-19 pandemic. Journal of Behavioral Addictions, 9(2), 184-186. https://doi.org/10.1556/2006.2020.00016

Király, O., Potenza, M. N., Stein, D. J., King, D. L., Hodgins, D. C., Saunders, J. B., Griffiths, M. D., Gjoneska, B., Billieux, J., Brand, M., Abbott, M. W., Chamberlain, S. R., Corazza, O., Burkauskas, J., Sales, C. M. D., Montag, C., Lochner, C., Grünblatt, E., Wegmann, E., ... Demetrovics, Z. (2020). Preventing problematic internet use during the COVID-19 pandemic: Consensus guidance. Comprehensive Psychiatry, 100, Article 152180. https://doi.org/10.1016/j.comppsych.2020.152180

Ko, C.-H., \& Yen, J.-Y. (2020). Impact of COVID-19 on gaming disorder: Monitoring and prevention. Journal of Behavioral Addictions, 9(2), 187-189. https://doi.org/10.1556/2006.2020.00040

Li, Y.-Y., Sun, Y., Meng, S.-Q., Bao, Y.-P., Cheng, J.-L., Chang, X.-W., Ran, M.-S., Sun, Y.-K., Kosten, T., Strang, J., Lu, L., \& Shi, J. (2021). Internet addiction increases in the general population during COVID-19: Evidence from China. The American Journal on Addictions, 30(4), 389-397. https://doi.org/10.1111/ajad.13156
Marston, H. R., \& Kowert, R. (2020). What role can videogames play in the COVID-19 pandemic? Emerald Open Research, 2, Article 34. https:// doi.org/10.35241/emeraldopenres.13727.2

McHugh, M. L. (2012). Interrater reliability: The kappa statistic. Biochemia Medica, 22(3), 276-282. https://doi.org/10.11613/BM.2012.031

Orben, A., Weinstein, N., \& Przybylski, A. K. (2020). Only Holistic and iterative change will fix digital technology research. Psychological Inquiry, 31(3), 235-241. https://doi.org/10.1080/1047840X.2020.1820221

Parry, D. A., Davidson, B. I., Sewall, C. J. R., Fisher, J. T., Mieczkowski, H., \& Quintana, D. S. (2021). A systematic review and meta-analysis of discrepancies between logged and self-reported digital media use. Nature Human Behaviour. https://doi.org/10.1038/s41562-021-01117-5

Poletti, M., \& Raballo, A. (2020). Letter to the editor: Evidence on school closure and children's social contact: Useful for coronavirus disease (COVID-19)? Eurosurveillance, 25(17), Article 2000758. https:// doi.org/10.2807/1560-7917.ES.2020.25.17.2000758

Raabe, I. J., Ehlert, A., Johann, D., \& Rauhut, H. (2020). Correction: Satisfaction of scientists during the COVID-19 pandemic lockdown. Humanities and Social Sciences Communications, 7(1), Article 168 https://doi.org/10.1057/s41599-020-00675-9

R Core Team. (2019). R: A language and environment for statistical computing. R Foundation for Statistical Computing. https://www.R-project.org/

Rome, O., Sinai, L., Sevitt, R., Meroody, A., Nadolne, M., Shilco, P., Jacobs, G. P., Shterenshis, M., Sosnow, A., Foonberg, M., Faridnia, E., Emrani, A., Hiekali, L., Shohed, C., Golshan, T., Roitblat, Y., Burger, J., Vaiman, M., Nehuliaieva, L., ... Shterenshis, M. (2021). Owls and larks do not exist: COVID-19 quarantine sleep habits. Sleep Medicine, 77, 177-183. https:// doi.org/10.1016/j.sleep.2020.09.003

Rudnicka, A., Newbold, J. W., Cook, D., Cecchinato, M. E., Gould, S. J. J., \& Cox, A. L. (2020). Eworklife: Developing effective strategies for remote working during the COVID-19 pandemic. https://www.microsoft.com/ en-us/research/publication/eworklife-developing-effective-strategies-forremote-working-during-the-covid-19-pandemic/

Ryan, R. M., Rigby, C. S., \& Przybylski, A. (2006). The motivational pull of video games: A self-determination theory approach. Motivation and Emotion, 30(4), 344-360. https://doi.org/10.1007/s11031-006-9051-8

Stieg, C. (2020, March 24). Why video games like The Sims and Animal Crossing are so soothing right now. CNBC. https://www.cnbc.com/2020/03/24/ psychological-benefits-of-the-sims-animal-crossing-during-covid-19.html

Teng, Z., Pontes, H. M., Nie, Q., Griffiths, M. D., \& Guo, C. (2021). Depression and anxiety symptoms associated with internet gaming disorder before and during the COVID-19 pandemic: A longitudinal study. Journal of Behavioral Addictions, 10(1), 169-180. https://doi.org/10 1556/2006.2021.00016

Vuorre, M., Zendle, D., Petrovskaya, E., Ballou, N., \& Przybylski, A. K. (2020). A large-scale study of changes to the quantity, quality, and distribution of video game play during the COVID-19 pandemic. https://doi.org/10.17605/OSF.IO/YA9JT

Wood, S. N. (2017). Generalized additive models: An introduction with $R$ (2nd ed.). CRC Press. https://doi.org/10.1201/9781315370279

Wood, S. N. (2020). mgcv: Mixed GAM computation vehicle with automatic smoothness estimation (Version 1.8-33) [Computer software]. https:// CRAN.R-project.org $/$ package $=\mathrm{mgcv}$

Zendle, D., Meyer, R., \& Ballou, N. (2020). The changing face of desktop video game monetisation: An exploration of exposure to loot boxes, pay to win, and cosmetic microtransactions in the most-played Steam games of 2010-2019. PLOS ONE, 15(5), Article e0232780. https://doi.org/10 .1371/journal.pone. 0232780

Received March 4, 2021

Revision received May 24, 2021

Accepted July 2, 2021 